\title{
Origin of Hierarchical Structures of Quark and Lepton Mass Matrices
}

\author{
Yoshio Koide $^{a}$ and Hiroyuki Nishiura ${ }^{b}$ \\ ${ }^{a}$ Department of Physics, Osaka University, Toyonaka, Osaka 560-0043, Japan \\ E-mail address: koide@kuno-g.phys.sci.osaka-u.ac.jp \\ ${ }^{b}$ Faculty of Information Science and Technology, Osaka Institute of Technology, Hirakata, \\ Osaka 573-0196, Japan \\ E-mail address: hiroyuki.nishiura@oit.ac.jp
}

\begin{abstract}
It is shown that the so-called "Yukawaon" model can give a unified description of masses, mixing and $C P$ violation parameters of quarks and leptons without using any hierarchical (family number-dependent) parameters besides the charged lepton masses. Here, we have introduced a phase matrix $P=\operatorname{daig}\left(e^{i \phi_{1}}, e^{i \phi_{2}}, e^{i \phi_{3}}\right)$ with the phase parameters $\left(\phi_{1}, \phi_{2}, \phi_{3}\right)$ which are described in terms of family number-independent parameters, together with using only the charged lepton mass parameters as the family number-dependent parameters. In this paper, the $C P$ violating phase parameters $\delta_{C P}^{q}$ and $\delta_{C P}^{\ell}$ in the standard expression of $V_{C K M}$ and $U_{P M N S}$ are predicted as $\delta_{C P}^{q} \simeq 72^{\circ}$ and $\delta_{C P}^{\ell} \simeq-76^{\circ}$, respectively, i.e. $\delta_{C P}^{\ell} \sim-\delta_{C P}^{q}$.
\end{abstract}

PCAC numbers: 11.30.Hv, 12.15.Ff, 14.60.Pq, 12.60.-i,

\section{Introduction}

\subsection{What is the Yukawaon model}

One of the big subjects in the particle physics is to investigate the origin of flavors. There is an attractive idea that the flavor physics is understood from the point of view of a family symmetry [1]. However, the symmetry has to be explicitly broken by the Yukawa coupling constants $Y_{f}(f=\nu, e, u, d)$ if we suppose the family symmetry to be a continuous symmetry. Therefore, the symmetry is usually considered as a discrete symmetry. If we adhere to the basic idea that the flavor symmetry should be a continuous symmetry which is unbroken at the start, we are forced to consider that the Yukawa coupling constants are effective coupling constants $Y_{f}^{e f f}$ which are given by vacuum expectation values (VEVs) of scalars ("Yukawaons") $Y_{f}$ with $3 \times 3$ components [2]:

$$
\left(Y_{f}^{e f f}\right)_{i}^{j}=\frac{y_{f}}{\Lambda}\left\langle Y_{f}\right\rangle_{i}^{j} \quad(f=u, d, \nu, e),
$$

where $\Lambda$ is an energy scale of the effective theory. In the Yukawaon model, all the flavons [3] are expressed by $3 \times 3$ components of $\mathrm{U}(3)$. We consider no substructures of it such as $2 \times 2$ and so on. 
In the Yukawaon model, we assume a U(3) family symmetry, and take the following wouldbe Yukawa interactions:

$$
\begin{gathered}
H_{Y}=\frac{y_{\nu}}{\Lambda}\left(\bar{\ell}_{L}\right)^{i}\left(\hat{Y}_{\nu}\right)_{i}^{j}\left(\nu_{R}\right)_{j} H_{u}+\frac{y_{e}}{\Lambda}\left(\bar{\ell}_{L}\right)^{i}\left(\hat{Y}_{e}\right)_{i}{ }^{j}\left(e_{R}\right)_{j} H_{d}+y_{R}\left(\bar{\nu}_{R}\right)^{i}\left(Y_{R}\right)_{i j}\left(\nu_{R}^{c}\right)^{j} \\
+\frac{y_{u}}{\Lambda}\left(\bar{q}_{L}\right)^{i}\left(\hat{Y}_{u}\right)_{i}{ }^{j}\left(u_{R}\right)_{j} H_{u}+\frac{y_{d}}{\Lambda}\left(\bar{q}_{L}\right)^{i}\left(\hat{Y}_{d}\right)_{i}{ }^{j}\left(d_{R}\right)_{j} H_{d},
\end{gathered}
$$

where $\ell_{L}=\left(\nu_{L}, e_{L}\right)$ and $q_{L}=\left(u_{L}, d_{L}\right)$ are $\mathrm{SU}(2)_{L}$ doublets. $H_{u}$ and $H_{d}$ are two Higgs doublets. The third term in Eq.(1.2) leads to the so-called neutrino seesaw mass matrix [4] $M_{\nu}=\hat{Y}_{\nu} Y_{R}^{-1} \hat{Y}_{\nu}^{T}$, where $\hat{Y}_{\nu}$ and $Y_{R}$ correspond to the Dirac and Majorana mass matrices of neutrinos, respectively. Hereafter, for convenience, we use notation $\hat{A}, A$ and $\bar{A}$ for fields with $\mathbf{8}+\mathbf{1}, \mathbf{6}$ and $\mathbf{6}^{*}$ of $\mathrm{U}(3)$, respectively.

In order to distinguish each Yukawaon from the others, we assume that $\hat{Y}_{f}$ have different $R$ charges from each other by considering $R$-charge conservation [a global $\mathrm{U}(1)$ symmetry in $N=1$ supersymmetry (SUSY)]. Of course, the $R$-charge conservation is broken at an energy scale $\Lambda$, at which the $\mathrm{U}(3)$ family symmetry is broken. For $R$ parity assignments, we inherit those in the standard SUSY model, i.e. $R$ parities of yukawaons $\hat{Y}_{f}$ (and all flavons) are the same as those of Higgs particles (i.e. $P_{R}$ (fermion) $=-1$ and $P_{R}$ (scalar $)=+1$ ), while quarks and leptons are assigned to $P_{R}$ (fermion) $=+1$ and $P_{R}($ scalar $)=-1$.

A remarkable characteristic of the Yukawaon model is that it is possible to understand the observed hierarchical structures of masses and mixings of quarks and leptons without using any family number-dependent parameters except for the charged lepton masses. That is, all the quark and lepton masses and mixings can be understood in terms of only the observed charged lepton masses. In the Yukawaon model so far, our aim seems to be almost accomplished except for the following problem only: Namely, we have obliged to introduce a phase matrix $\langle P\rangle=\operatorname{diag}\left(e^{i \phi_{1}}, e^{i \phi_{2}}, e^{i \phi_{3}}\right)$ in order to give a good fitting for the Cabibbo-Kobayashi-Maskawa (CKM) mixing matrix [5], where $\left(\phi_{1}, \phi_{2}, \phi_{3}\right)$ have been introduced as family number-dependent parameters. In this paper, however, we will relate those parameters $\left(\phi_{1}, \phi_{2}, \phi_{3}\right)$ to the observed charged lepton masses $m_{e i}$ as discussed in Sec.4.

Relations among Yukawaon VEVs $\left\langle\hat{Y}_{f}\right\rangle$ are obtained by supersymmetric vacuum conditions from $\mathrm{U}(3)$ symmetric and $R$-charge conserved superpotential. In this article, the Yukawaon $\operatorname{VEVs}\left\langle\hat{Y}_{f}\right\rangle$ are related to VEVs of fundamental flavons $\Phi_{f}$ with a common bilinear form to all flavors: 1

$$
\left\langle\hat{Y}_{f}\right\rangle=k_{f}\left\langle\Phi_{f}\right\rangle\left\langle\bar{\Phi}_{f}\right\rangle+\xi_{f} \mathbf{1} \quad(f=u, d, \nu, e),
$$

\footnotetext{
${ }^{1}$ In earlier Yukawaon models [6], the bilinear form was only for the up-quark sector, and the model could excellently lead to the so-called tribimaximal mixing [7] in the lepton mixing under the use of only a few parameters. However, the model [6] (and also [8]) could not give the observed sizable mixing angle $\theta_{13}$. We found that we can give the observed value $\sin ^{2} 2 \theta_{13} \simeq 0.1$ [9] only when we consider that all VEV relations are given by a common bilinear form Eq.(1.3) [10].
} 
where $\mathbf{1}=\operatorname{diag}(1,1,1)$. Here, the VEV matrices $\left\langle\Phi_{f}\right\rangle$ and $\left\langle\bar{\Phi}_{f}\right\rangle$ are commonly related to a fundamental flavon VEVs $\left\langle\Phi_{0}\right\rangle$ and $\left\langle\bar{\Phi}_{0}\right\rangle$ by

$$
\begin{aligned}
& \left\langle\bar{P}_{f}\right\rangle^{i k}\left\langle\Phi_{f}\right\rangle_{k l}\left\langle\bar{P}_{f}\right\rangle^{l j}=k_{f}^{\prime}\left\langle\bar{\Phi}_{0}\right\rangle^{i \alpha}\left\langle S_{f}\right\rangle_{\alpha \beta}\left\langle\bar{\Phi}_{0}\right\rangle^{\beta j}, \quad(f=u, d, \nu, e) . \\
& \left\langle P_{f}\right\rangle_{i k}\left\langle\bar{\Phi}_{f}\right\rangle^{k l}\left\langle P_{f}\right\rangle_{l j}=k_{f}^{\prime}\left\langle\Phi_{0}\right\rangle_{i \alpha}\left\langle\bar{S}_{f}\right\rangle^{\alpha \beta}\left\langle\Phi_{0}\right\rangle_{\beta j},
\end{aligned}
$$

where $i$ and $\alpha$ are indices of $\mathrm{U}(3)$ and $\mathrm{U}(3)^{\prime}$, respectively. For the VEV structures of $P_{f}$ and $\bar{P}_{f}$ in Eq.(1.4) and the $\xi_{f}$ terms in Eq.(1.3), we discuss in the next section.

The VEV structures $\left\langle S_{f}\right\rangle$ and $\left\langle\bar{S}_{f}\right\rangle$ in Eq.(1.4) are given by 2

$$
\left\langle S_{f}\right\rangle=\left\langle\bar{S}_{f}\right\rangle=\mathbf{1}+a_{f} X_{3},
$$

where

$$
\mathbf{1}=\left(\begin{array}{lll}
1 & 0 & 0 \\
0 & 1 & 0 \\
0 & 0 & 1
\end{array}\right), \quad X_{3}=\frac{1}{3}\left(\begin{array}{ccc}
1 & 1 & 1 \\
1 & 1 & 1 \\
1 & 1 & 1
\end{array}\right)
$$

The form of Eq.(1.6) is understood by a symmetry breaking $\mathrm{U}(3)^{\prime} \rightarrow \mathrm{S}_{3}$.

\subsection{Charged lepton sector as a fundamental flavor basis}

We consider that the charged lepton mass matrix is the most fundamental one compared with other mass matrices and that the charged lepton mass values play an essential role in understanding the flavor physics. The points of our postulation are as follows:

(i) There is a fundamental flavon $\Phi_{0}$, and the reference basis in the flavor physics is defined by the diagonal basis of $\left\langle\Phi_{0}\right\rangle$ and $\left\langle\bar{\Phi}_{0}\right\rangle$ :

$$
\left\langle\Phi_{0}\right\rangle=\left\langle\bar{\Phi}_{0}\right\rangle \equiv v_{0} \operatorname{diag}\left(x_{1}, x_{2}, x_{3}\right) .
$$

where $x_{i}$ are real parameters with $x_{1}^{2}+x_{2}^{2}+x_{3}^{3}=1$.

(ii) In the reference basis, the $\mathrm{U}(3)^{\prime}$ family symmetry is broken into $\mathrm{S}_{3}$, i.e. VEVs of flavons $S_{f}$ and $\bar{S}_{f}$ take the form (1.5).

(iii) The charged lepton mass matrix $\left\langle\hat{Y}_{e}\right\rangle$ should be diagonal and real as well as $\left\langle\Phi_{0}\right\rangle$ and $\left\langle\bar{\Phi}_{0}\right\rangle$, and it should be described only in terms of the fundamental parameters $x_{i}$. Therefore, with demanding simplicity too, we require

$$
a_{e}=0, \quad \xi_{e}=0 .
$$

This means $x_{i} \propto m_{e i}^{1 / 4}\left(m_{e i}=\left(m_{e}, m_{\mu}, m_{\tau}\right)\right)$. In Sec.3, we use the following parameter values of $x_{i}$,

$$
\left(x_{1}, x_{2}, x_{3}\right)=(0.115144,0.438873,0.891141) .
$$

\footnotetext{
${ }^{2}$ The form (1.5) was suggested by a "democratic universal seesaw" mass matrix model [11, in which quark mass matrices are given by a form $\left\langle\Phi_{e}\right\rangle\left(\mathbf{1}+a_{f} X_{3}\right)\left\langle\Phi_{e}\right\rangle$.
} 
In (1.9), we have used running mass values $m_{e}(\mu)=0.000486847 \mathrm{GeV}, m_{\mu}(\mu)=0.102751$ $\mathrm{GeV}$ and $m_{\tau}(\mu)=1.7467 \mathrm{GeV}$ as the charged lepton mass values at $\mu=M_{Z}$, because our numerical predictions in the quark mass ratios are done at $\mu=M_{Z}$. Note that the mass values $\left(m_{e}, m_{\mu}, m_{\tau}\right)$ have a large hierarchical structure, i.e. $m_{e} / m_{\tau} \sim 10^{-4}$, while the values (1.9) have a mild hierarchical structure, i.e. $x_{1} / x_{3} \sim 10^{-1}$.

In this paper, we do not ask the origin of the value $\left(x_{1}, x_{2}, x_{3}\right)$. However, for reference, in Appendices A and B, we will demonstrate an example of the charged lepton mass relation in the present Yukawaon model.

\subsection{What is new}

The new characteristic points of the present Yukawaon model are as follows:

(i) The bilinear form of the Yukawaon VEVs given in Eqs.(1.3) and (1.4) has been adopted for some flavor sectors in the previous paper [10], too. However, in the present paper, we apply it to all the sectors $f=u, d, \nu, e$.

(ii) So far, we have needed a VEV matrix $P$ given by

$$
P=\operatorname{diag}\left(e^{i \phi_{1}}, e^{i \phi_{2}}, e^{i \phi_{3}}\right),
$$

in order to fit CKM mixing matrix $V_{C K M}$ reasonably. However, our aim was to describe all the masses and mixing of quarks and leptons in terms of family number-independent parameters expect for the charged lepton masses. Therefore, the phase parameters $\phi_{i}$ were against our aim and unwelcome as it is. In the present paper, we try to denote the family number-dependent parameters $\phi_{i}$ in terms of the observed charged lepton masses $m_{e i}$. The details are discussed in Sec.4.

(iii) In general, the phase matrix $P$ affects not only the CKM quark mixing matrix $V_{C K M}$ but also the Pontecorvo-Maki-Nakagawa-Sakata [12] (PMNS) lepton mixing matrix $U_{P M N S}$. Furthermore, predicted value of the $C P$ violating phase parameter $\delta_{C P}^{\ell}$ in $U_{P M N S}$ will depend on an appearing position of $P$ which is arbitrary at present. In the present paper, as we will discuss in Sec.2, we construct a model in which the phase matrix $P$ appears in the up-quark sector only, and it affects not only the $V_{C K M}$ but also the $U_{P M N S}$ through Eq.(2.5) as we give later. As a result, as we discuss in Sec.3, we predict $C P$ violation phase parameters $\delta_{C P}^{q}$ and $\delta_{C P}^{\ell}$ in the standard expression of $V_{C K M}$ and $U_{P M N S}$ as $\delta_{C P}^{\ell} \simeq-\delta_{C P}^{q} \simeq-70^{\circ}$ unlike the previous papers.

In Sec.2, we construct a mass matrix model base on a Yukawaon model in which the phase matrix $P$ appears in the up-quark sector. In Sec.3, parameter fitting are discussed. Especially, it is shown that $C P$ violating phase parameters $\delta_{C P}^{q}$ and $\delta_{C P}^{\ell}$ in the standard expression of $V_{C K M}$ and $U_{P M N S}$ are predicted as $\delta_{C P}^{q} \simeq 72^{\circ}$ and $\delta_{C P}^{\ell} \simeq-76^{\circ}$, respectively, i.e. $\delta_{C P}^{\ell} \sim-\delta_{C P}^{q}$. In Sec.4, we will propose a new relation between $P$ and $m_{e i}$. Finally, Sec.5 is devoted to concluding remarks. In Appendices A and B, we will demonstrate an example of the charged lepton mass relation in the present Yukawaon model. 


\section{Model}

\subsection{VEV relations and superpotentials}

The VEV relations of Yukawaons have already been given in Eqs.(1.3) and (1.4) in Sec.1. Those VEV relations are derived from a $\mathrm{U}(3) \times \mathrm{U}(3)^{\prime}$ symmetric superpotential. For example, for the VEV relation (1.3), we assume [10]

$$
W_{Y}=\sum_{f=u, d, \nu, e}\left\{\operatorname{Tr}\left[\left(\mu_{f} \hat{Y}_{f}+\lambda_{f} \Phi_{f} \bar{\Phi}_{f}\right) \hat{\Theta}_{f}\right]+\operatorname{Tr}\left[\mu_{f}^{\prime} \hat{Y}_{f}+\lambda_{f}^{\prime} \Phi_{f} \bar{\Phi}_{f}\right] \operatorname{Tr}\left[\hat{\Theta}_{f}\right]\right\}
$$

The supersymmetric vacuum condition $\partial W / \partial \hat{\Theta}_{f}=0$ leads to

$$
\mu_{f} \hat{Y}_{f}+\lambda_{f} \Phi_{f} \bar{\Phi}_{f}+\operatorname{Tr}\left[\mu_{f}^{\prime} \hat{Y}_{f}+\lambda_{f}^{\prime} \Phi_{f} \bar{\Phi}_{f}\right] \mathbf{1}=0
$$

that is, to the VEV relation (1.3) with the $\xi_{f}$ term,

$$
\xi_{f}=\frac{\mu_{f}^{\prime} \lambda_{f}-\mu_{f} \lambda_{f}^{\prime}}{\left(\mu_{f}+3 \mu_{f}^{\prime}\right) \mu_{f}} \operatorname{Tr}\left[\Phi_{f} \bar{\Phi}_{f}\right]
$$

Here, we assume that flavons $\hat{\Theta}_{f}$ always take $\left\langle\hat{\Theta}_{f}\right\rangle=0$, so that vacuum conditions, which are obtained by differentiating the superpotential $W_{Y}$ with respect to other flavons, do not give any additional VEV relations, because those relations always include one $\left\langle\hat{\Theta}_{f}\right\rangle$.

In a similar way, we can obtain the VEV relation (1.4) by assuming

$$
W_{\Phi}=\sum_{f=u, d, \nu, e}\left(\lambda_{f 1}\left(\bar{P}_{f}\right)^{i k}\left(\Phi_{f}\right)_{k l}\left(\bar{P}_{f}\right)^{l j}+\lambda_{f 2}\left(\bar{\Phi}_{0}\right)^{i \alpha}\left(S_{f}\right)_{\alpha \beta}\left(\bar{\Phi}_{0}\right)^{\beta j}\right)\left(\Theta_{f}\right)_{i j},
$$

and so on. Although we assumed a tad pole term $\operatorname{Tr}\left[\hat{\Theta}_{f}\right]$ in $(2.1)$, we consider that such a term is a special case only for $\hat{\Theta}_{f}$ with $(\mathbf{8}+\mathbf{1})$ of $\mathrm{U}(3)$. Therefore, such $\xi_{f}$ terms do not appear in the relation (1.4).

In order to give a neutrino mass matrix with seesaw mechanism, $M_{\nu}=k_{\nu}\left\langle\hat{Y}_{\nu}^{T}\right\rangle\left\langle Y_{R}\right\rangle^{-1}\left\langle\hat{Y}_{\nu}\right\rangle$, correspondingly to the following Majorana neutrino mass matrix $M_{R}$ (i.e. $\left\langle Y_{R}\right\rangle$ ), we assume VEV matrix relation

$$
\left\langle Y_{R}\right\rangle=\left\langle\hat{Y}_{e}\right\rangle\left\langle\Phi_{u}\right\rangle+\left\langle\Phi_{u}\right\rangle\left\langle\hat{Y}_{e}^{T}\right\rangle
$$

according to the previous Yukawaon model [10].

\subsection{VEV structures of $P_{f}$}

Prior to discussing VEV forms $\left\langle P_{f}\right\rangle$ and $\left\langle\bar{P}_{f}\right\rangle$ given in Eq.(1.4), let us consider the following superpotential

$$
W_{P}=\frac{\lambda_{1}}{\Lambda} \operatorname{Tr}[P \bar{P} E \bar{E}]+\frac{\lambda_{2}}{\Lambda} \operatorname{Tr}[P \bar{P}] \operatorname{Tr}[E \bar{E}]
$$


where, in order to distinguish $P$ from $E$, we assign $R$ charges of $P$ and $E$ as

$$
R(P)=R(\bar{P})=\frac{1}{2}(1-\Delta), \quad R(E)=R(\bar{E})=\frac{1}{2}(1+\Delta)
$$

so that $R(P)+R(\bar{P})+R(E)+R(\bar{E})=2$. The supersymmetric vacuum conditions lead to

$$
\langle P\rangle\langle\bar{P}\rangle=\mathbf{1}, \quad\langle E\rangle\langle\bar{E}\rangle=\mathbf{1}
$$

We define specific solutions of (2.8) as

$$
\langle P\rangle=\operatorname{diag}\left(e^{i \phi_{1}}, e^{i \phi_{2}}, e^{i \phi_{3}}\right), \quad\langle E\rangle=\operatorname{diag}(1,1,1) .
$$

We consider that VEV of each flavon $\left\langle P_{f}\right\rangle$ given in Eq.(1.4) is given by either $\langle P\rangle$ or $\langle E\rangle$ in Eq.(2.9) under the $D$-term condition as discussed in (2.10) below.

On the other hand, let us recall that, in general, VEV matrix $\langle\bar{A}\rangle$ is related to VEV matrix $\langle A\rangle$ under the $D$ term condition as

$$
\langle\bar{A}\rangle=\langle A\rangle^{*}, \quad \text { or } \quad\langle\bar{A}\rangle=\langle A\rangle
$$

Let us back to the relations (1.4). We take

$$
\begin{gathered}
\left\langle\Phi_{0}\right\rangle=\left\langle\bar{\Phi}_{0}\right\rangle=\operatorname{diag}\left(x_{1}, x_{2} \cdot x_{3}\right), \\
\left\langle S_{f}\right\rangle=\left\langle\bar{S}_{f}\right\rangle=\mathbf{1}+a_{f} e^{i \alpha_{f}} X_{3},
\end{gathered}
$$

while

$$
\langle\bar{P}\rangle=\langle P\rangle^{*}=\operatorname{diag}\left(e^{-i \phi_{1}}, e^{-i \phi_{2}}, e^{-i \phi_{3}}\right),
$$

where parameters $x_{i}, \phi_{i}, a_{f}$ and $\alpha_{f}$ are real. Then, according as $\left\langle\bar{\Phi}_{f}\right\rangle=\left\langle\Phi_{f}\right\rangle^{*}$ or $\left\langle\bar{\Phi}_{f}\right\rangle=\left\langle\Phi_{f}\right\rangle$, the relations (1.4) require $\left\langle\bar{P}_{f}\right\rangle=\left\langle P_{f}\right\rangle^{*}$ or $\left\langle\bar{P}_{f}\right\rangle=\left\langle P_{f}\right\rangle$. For example, when we take the case $\left\langle\bar{\Phi}_{f}\right\rangle=\left\langle\Phi_{f}\right\rangle^{*}$, Eq.(1.4) becomes

$$
\begin{aligned}
& \left\langle P_{f}\right\rangle^{*}\left\langle\Phi_{f}\right\rangle\left\langle P_{f}\right\rangle^{*}=\left\langle\Phi_{0}\right\rangle\left\langle S_{f}\right\rangle\left\langle\Phi_{0}\right\rangle, \\
& \left\langle P_{f}\right\rangle\left\langle\Phi_{f}\right\rangle^{*}\left\langle P_{f}\right\rangle=\left\langle\Phi_{0}\right\rangle\left\langle S_{f}\right\rangle\left\langle\Phi_{0}\right\rangle .
\end{aligned}
$$

Two equations in (2.14) cannot simultaneously satisfied without $\alpha_{f}=0$. On the other hand, when $\left\langle\bar{\Phi}_{f}\right\rangle=\left\langle\Phi_{f}\right\rangle$, Eq.(1.4) becomes

$$
\begin{aligned}
& \left\langle P_{f}\right\rangle^{*}\left\langle\Phi_{f}\right\rangle\left\langle P_{f}\right\rangle^{*}=\left\langle\Phi_{0}\right\rangle\left\langle S_{f}\right\rangle\left\langle\Phi_{0}\right\rangle, \\
& \left\langle P_{f}\right\rangle\left\langle\Phi_{f}\right\rangle\left\langle P_{f}\right\rangle=\left\langle\Phi_{0}\right\rangle\left\langle S_{f}\right\rangle\left\langle\Phi_{0}\right\rangle .
\end{aligned}
$$


Then, for the case of $\alpha_{f} \neq 0$, the two equations (2.15) are satisfied only when $\phi_{i}=0$ [i.e. $\left\langle P_{f}\right\rangle=\langle E\rangle$. As a result, we consider only two cases: (i) for the case of $\alpha_{f} \neq 0$, we regard $\left\langle P_{f}\right\rangle$ as $\langle E\rangle$ given in (2.9), and (ii) only for the case of $\alpha_{f}=0$, we regard $\left\langle P_{f}\right\rangle$ as $\langle P\rangle$ with $\phi_{i} \neq 0$.

The parameters $\alpha_{f}$ affect not only $C P$ violation, but also mass ratios. In the up-quark sector, as we discuss in Sec.3, we can fit up-quark mass ratios $m_{u} / m_{c}$ and $m_{c} / m_{t}$ by taking two parameters $a_{u}$ and $\xi_{u}$ (keeping $\alpha_{u}=0$ ). Therefore, we regard the up-quark sector as the case of $\alpha_{f}=0$, so that we regard $\left\langle P_{f}\right\rangle$ as $\left\langle P_{f}\right\rangle=\langle P\rangle$. Also, since we have taken $a_{e}=0$, we have to regard $\left\langle P_{e}\right\rangle$ as $\left\langle P_{e}\right\rangle=\langle P\rangle$. Note that $\langle P\rangle$ and $\left\langle\hat{Y}_{e}\right\rangle$ are diagonal, so that they are commutable each other. Therefore, $\left\langle P_{e}\right\rangle$ does not play any essential physical role in the parameter fitting of the masses and mixing of quarks. Hereafter, we denote $\left\langle P_{e}\right\rangle$ as $\langle E\rangle$ from the practical point of view, except for a case of counting of $R$ charge. On the other hand, in down-quark sector, we cannot fit down-quark mass ratios $m_{d} / m_{s}$ and $m_{s} / m_{b}$ without help of $\alpha_{d} \neq 0$. Therefore, we regard down-quark sector as a case of $\left\langle P_{f}\right\rangle=\langle E\rangle$. Thus, we have the selection rule, $\left\langle P_{f}\right\rangle=\langle P\rangle$ or $\left\langle P_{f}\right\rangle=\langle E\rangle$, as a phenomenological one. For neutrino sector, we have no phenomenological information. For simplicity, we take a fewer parameter scheme $\left(\alpha_{\nu} \neq 0\right.$ rather than $\left.\phi_{i}^{\nu} \neq 0\right)$. Hereafter, we will use the notation $P_{f}$ as

$$
\begin{array}{ll}
P_{f}=P & \text { for } \quad f=u, e \\
P_{f}=E & \text { for } \quad f=d, \nu
\end{array}
$$

Sometimes, for convenience, we use notations $P_{u}, P_{e}$, and so on, although we identify $P_{u}$ and $P_{e}$ as one flavon $P$, and also $P_{d}$ and $P_{\nu}$ as one flavon $E$.

The phase matrix $\langle P\rangle$ does not affect mass ratios. Since $\left\langle P_{u}\right\rangle=\langle P\rangle$, the phase parameters affects $C P$ violation phase $\delta_{C P}^{q}$ in the CKM mixing matrix $V_{C K M}$. However, note that the phase in $\left\langle P_{u}\right\rangle=\langle P\rangle$ can also affect $C P$ violation phase $\delta_{C P}^{\ell}$ in the PMNS mixing matrix $U_{P M N S}$, because the phase in $P_{u}$ can affect $Y_{R}$ through $\Phi_{u}$ as shown in Eq.(2.5). This is the most notable point in the present paper 3

The details are discussed in the next section, Sec.3.

\section{$2.3 \quad R$ charge assignments}

In this model, the number of flavons is larger than that of VEV relations. Therefore, in general, we cannot uniquely determine $R$ charges of flavons. Since we demand to assign $R$ charges as simple as possible, we put the following rules for simplicity:

(i) We assign the same $R$ charge to flavons $A$ and $\bar{A}$ :

$$
R(A)=R(\bar{A}),
$$

\footnotetext{
${ }^{3}$ A similar model with $P_{u}$ has been discussed in Ref. [10. However, we has neglected a possible effect of $\left\langle P_{u}\right.$ in the neutrino mixing.
} 
independently whether $\langle\bar{A}\rangle=\langle A\rangle^{*}$ or $\langle\bar{A}\rangle=\langle A\rangle$. Then, we obtain $R$ charge relations

$$
R\left(\hat{Y}_{f}\right)=2 R\left(\Phi_{f}\right) \equiv 2 r_{f} \quad(f=u, d, \nu, e)
$$

and

$$
R\left(\Phi_{f}\right)=R\left(\bar{\Phi}_{f}\right)=R\left(S_{f}\right)+2 R\left(\Phi_{0}\right)-2 R\left(P_{f}\right) \quad(f=u, d, \nu, e)
$$

from Eqs.(1.3) and (1.4), and

$$
\begin{aligned}
& R\left(P_{u}\right)=R\left(P_{e}\right)=R(P) \equiv \frac{1}{2}(1+\Delta), \\
& R\left(P_{d}\right)=R\left(P_{\nu}\right)=R(E) \equiv \frac{1}{2}(1-\Delta),
\end{aligned}
$$

from Eqs.(2.16) and (2.7). Therefore, from Eq.(2.19), we obtain the following relations:

$$
\begin{aligned}
& R\left(\Phi_{e}\right)-R\left(S_{e}\right)=R\left(\Phi_{u}\right)-R\left(S_{u}\right)=2 R\left(\Phi_{0}\right)-(1+\Delta), \\
& R\left(\Phi_{\nu}\right)-R\left(S_{\nu}\right)=R\left(\Phi_{d}\right)-R\left(S_{d}\right)=2 R\left(\Phi_{0}\right)-(1-\Delta) .
\end{aligned}
$$

(ii) We can regard that $R$ charges of $\hat{Y}_{f}$ are determined only by those of the $\mathrm{SU}(2)_{L}$ singlet fermions $f^{c}$. Therefore, we simply assign

$$
R\left(\ell H_{u}\right)=R\left(\ell H_{d}\right)=R\left(q H_{u}\right)=R\left(q H_{d}\right) \equiv r_{H}+2 .
$$

Since those have different quantum number of $\mathrm{U}(1)_{Y}$, we can distinguish those from each other in spite of the relation (2.22). Then, we obtain a simple $R$ charge relation

$$
R\left(\hat{Y}_{f}\right)+R\left(f^{c}\right)=-r_{H}
$$

For $Y_{R}$, we obtain

$$
R\left(Y_{R}\right)=2-2 R\left(\nu^{c}\right)=2 r_{H}+2+2 R\left(\hat{Y}_{\nu}\right)
$$

from Eqs.(2.1) and (2.23). On the other hand, from Eq.(2.5), $R\left(Y_{R}\right)$ must be satisfied a relation

$$
R\left(Y_{R}\right)=R\left(\Phi_{u}\right)+2 R\left(\Phi_{e}\right)
$$

From Eqs.(2.24) and (2.25), we have the following constraint

$$
2 R\left(\Phi_{e}\right)-4 R\left(\Phi_{\nu}\right)+R\left(\Phi_{u}\right)=2 r_{H}+2
$$

Even under the these constraints, we cannot still completely fix the $R$ charges of whole flavons. In the present model, $R$ charge assignments are not so essential, so that it is enough to assign $\mathrm{R}$ charges to distinguish flavons with the same $\mathrm{U}(3)$ from each other. That is, we are satisfied with any $R$-charge numbers which satisfy the relations (2.18) - (2.26). Nevertheless, it 
is desirable to have explicit $R$-charge assignments as simple as possible. Therefore, let us go on our search for explicit $R$-charge assignments.

First, for simplicity, we put

$$
R\left(\Phi_{0}\right)=\frac{1}{2}
$$

Then. Eq.(2.21) becomes to be simpler relations

$$
\begin{array}{ll}
R\left(S_{f}\right)=R\left(\Phi_{f}\right)+\Delta & (f=e, u), \\
R\left(S_{f}\right)=R\left(\Phi_{f}\right)-\Delta \quad & (f=\nu, d) .
\end{array}
$$

Now, let us discuss possible $R$-charge assignments for Yukawaons $\hat{Y}_{f}$ under the conditions discussed above. If we have $R\left(\hat{Y}_{f}\right)=0$, then we can attach the field $\hat{Y}_{f}$ on any term in superpotential. Therefore, we require $R\left(\hat{Y}_{f}\right) \neq 0$ for any $f=e, \nu, d, u$. Also, we have to require $R\left(\hat{Y}_{f} \hat{Y}_{f^{\prime}}\right) \neq 0$ for any combination of $f$ and $f^{\prime}$. As a result, we have to consider that whole $R$ values of $\hat{Y}_{f}$ are positive. Furthermore, we speculate that the values of $R$ will be describe by simple integers. Of course, the $R$ charges have to satisfy the relation (2.26). Therefore, we assign simpler $R$ charges to the Yukawaons $\hat{Y}_{f}$ on trial as follows:

$$
\left(R\left(\hat{Y}_{e}\right), R\left(\hat{Y}_{u}\right), R\left(\hat{Y}_{\nu}\right), R\left(\hat{Y}_{d}\right)\right)=(1,2,3,4),
$$

that is,

$$
\left(R\left(\Phi_{e}\right), R\left(\Phi_{u}\right), R\left(\Phi_{\nu}\right), R\left(\Phi_{d}\right)\right)=\left(\frac{1}{2}, 1, \frac{3}{2}, 2\right)
$$

This assignment satisfies the condition (2.25) for $R\left(Y_{R}\right)$ with $r_{H}=-3$.

In Table 1 , as a summary of Sec.2, we present the assignments of $\mathrm{SU}(2)_{L} \times \mathrm{SU}(3)_{c} \times \mathrm{U}(3) \times \mathrm{U}(3)^{\prime}$ and the $R$ charges of the fields in the present model.

\section{Parameter fitting}

\subsection{How many parameters?}

Our mass matrices $Y_{f}$ for $f=e, \nu, d, u$ with the VEV relations discussed in Sec.1 and Sec.2 are summarized as follows:

$$
\begin{aligned}
Y_{e}=\Phi_{e} \Phi_{e}^{*}, & \\
& \Phi_{e}=P^{*} \Phi_{0} \Phi_{0} P^{*} \\
& \Phi_{e}^{*}=P \Phi_{0} \Phi_{0} P \\
& \Phi_{0}=\operatorname{diag}\left(x_{1}, x_{2}, x_{3}\right),
\end{aligned}
$$


Table 1: Assignments of $\mathrm{SU}(2)_{L} \times \mathrm{SU}(3)_{c} \times \mathrm{U}(3) \times \mathrm{U}(3)^{\prime}$. For $R$ charges, see subsection 2.3. We assign the same $R$ charges for flavons $A$ and $\bar{A}$, e.g. $R(A)=R(\bar{A})$. For a special choice, $r_{e}, r_{\nu}$, $r_{u}$ and $r_{d}$ are taken as $r_{e}=1 / 2, r_{\nu}=3 / 2, r_{u}=2 / 2$ and $r_{d}=4 / 2$.

\begin{tabular}{|c|cc|cc|cc|}
\hline & $\ell=(\nu, e)$ & $f^{c}=\nu^{c}, e^{c}$ & $q=(u, d)$ & $f^{c}=u^{c}, d^{c}$ & $H_{u}$ & $H_{d}$ \\
\hline $\mathrm{SU}(2)_{L}$ & $\mathbf{2}$ & $\mathbf{1}$ & $\mathbf{2}$ & $\mathbf{1}$ & $\mathbf{2}$ & $\mathbf{2}$ \\
$\mathrm{SU}(3)_{c}$ & $\mathbf{1}$ & $\mathbf{1}$ & $\mathbf{3}$ & $\mathbf{3}^{*}$ & $\mathbf{1}$ & $\mathbf{1}$ \\
\hline $\mathrm{U}(3)$ & $\mathbf{3}$ & $\mathbf{3}^{*}$ & $\mathbf{3}$ & $\mathbf{3}^{*}$ & $\mathbf{1}$ & $\mathbf{1}$ \\
$\mathrm{U}(3)^{\prime}$ & $\mathbf{1}$ & $\mathbf{1}$ & $\mathbf{1}$ & $\mathbf{1}$ & $\mathbf{1}$ & $\mathbf{1}$ \\
\hline$R$ & 2 & $-\left(2 r_{f}+r_{H}\right)$ & 2 & $-\left(2 r_{f}+r_{H}\right)$ & $r_{H}$ & $r_{H}$ \\
\hline
\end{tabular}

\begin{tabular}{|cc|cc|cc|cc|cc|}
\hline$\hat{Y}_{f}$ & $Y_{R}$ & $\bar{\Phi}_{f}$ & $\Phi_{f}$ & $\bar{\Phi}_{0}$ & $\bar{\Phi}_{0}$ & $S_{e, u}$ & $\bar{S}_{e, u}$ & $S_{\nu, d}$ & $\bar{S}_{\nu, d}$ \\
\hline $\mathbf{1}$ & $\mathbf{1}$ & $\mathbf{1}$ & $\mathbf{1}$ & $\mathbf{1}$ & $\mathbf{1}$ & $\mathbf{1}$ & $\mathbf{1}$ & $\mathbf{1}$ & $\mathbf{1}$ \\
$\mathbf{1}$ & $\mathbf{1}$ & $\mathbf{1}$ & $\mathbf{1}$ & $\mathbf{1}$ & $\mathbf{1}$ & $\mathbf{1}$ & $\mathbf{1}$ & $\mathbf{1}$ & $\mathbf{1}$ \\
\hline $\mathbf{8}+\mathbf{1}$ & $\mathbf{6}$ & $\mathbf{6}$ & $\mathbf{6}^{*}$ & $\mathbf{3}$ & $\mathbf{3}^{*}$ & $\mathbf{1}$ & $\mathbf{1}$ & $\mathbf{1}$ & $\mathbf{1}$ \\
$\mathbf{1}$ & $\mathbf{1}$ & $\mathbf{1}$ & $\mathbf{1}$ & $\mathbf{3}$ & $\mathbf{3}^{*}$ & $\mathbf{6}$ & $\mathbf{6}^{*}$ & $\mathbf{6}$ & $\mathbf{6}^{*}$ \\
\hline $2 r_{f}$ & $r_{R}$ & \multicolumn{2}{|c|}{$r_{f}$} & \multicolumn{2}{|c|}{$1 / 2$} & \multicolumn{2}{|c|}{$r_{e, u}-\Delta$} & \multicolumn{2}{|c|}{$r_{\nu, d}+\Delta$} \\
\hline
\end{tabular}

\begin{tabular}{|cccc|c|c|cc|}
\hline$P$ & $\bar{P}$ & $E$ & $\bar{E}$ & $\hat{\Theta}_{f}$ & $\bar{\Theta}_{R}$ & $\Theta_{\Phi f}$ & $\bar{\Theta}_{\Phi f}$ \\
\hline $\mathbf{1}$ & $\mathbf{1}$ & $\mathbf{1}$ & $\mathbf{1}$ & $\mathbf{1}$ & $\mathbf{1}$ & $\mathbf{1}$ & $\mathbf{1}$ \\
$\mathbf{1}$ & $\mathbf{1}$ & $\mathbf{1}$ & $\mathbf{1}$ & $\mathbf{1}$ & $\mathbf{1}$ & $\mathbf{1}$ & $\mathbf{1}$ \\
\hline $\mathbf{6}$ & $\mathbf{6}^{*}$ & $\mathbf{6}$ & $\mathbf{6}^{*}$ & $\mathbf{8}+\mathbf{1}$ & $\mathbf{6}^{*}$ & $\mathbf{6}$ & $\mathbf{6}^{*}$ \\
$\mathbf{1}$ & $\mathbf{1}$ & $\mathbf{1}$ & $\mathbf{1}$ & $\mathbf{1}$ & $\mathbf{1}$ & $\mathbf{1}$ & $\mathbf{1}$ \\
\hline$\frac{1}{2}(1+\Delta)$ & $\frac{1}{2}(1-\Delta)$ & $2-2 r_{f}$ & $2-r_{R}$ & $1-R\left(S_{f}\right)$ \\
\hline
\end{tabular}




$$
\begin{aligned}
& Y_{\nu}=\Phi_{\nu} \Phi_{\nu}+\xi_{\nu} \mathbf{1}, \\
& \Phi_{\nu}=E \Phi_{0}\left(\mathbf{1}+a_{\nu} e^{i \alpha_{\nu}} X_{3}\right) \Phi_{0} E, \\
& Y_{u}=\Phi_{u} \Phi_{u}^{*}+\xi_{u} \mathbf{1}, \\
& \\
& \Phi_{u}=P^{*} \Phi_{0}\left(\mathbf{1}+a_{u} X_{3}\right) \Phi_{0} P^{*}, \\
& \Phi_{u}^{*}=P \Phi_{0}\left(\mathbf{1}+a_{u} X_{3}\right) \Phi_{0} P, \\
& Y_{d}=\Phi_{d} \Phi_{d}+\xi_{d} \mathbf{1}, \\
& \Phi_{d}=E \Phi_{0}\left(\mathbf{1}+a_{d} e^{i \alpha_{d}} X_{3}\right) \Phi_{0} E,
\end{aligned}
$$

Neutrino mass matrix with seesaw mechanism is given by

$$
M_{\nu}=Y_{\nu} Y_{R}^{-1} Y_{\nu}, \quad Y_{R}=Y_{e} \Phi_{u}+\Phi_{u} Y_{e},
$$

Note that $\Phi_{e}^{*}$ in Eq.(3.1) and $\Phi_{u}^{*}$ in Eq.(3.3) are not $\Phi_{e}$ and $\Phi_{u}$, respectively. Here, for convenience, we have dropped the notations "〈" and "〉". We also make no distinction of property under $\mathrm{U}(3) \times \mathrm{U}(3)^{\prime}$, i.e. we denote $\hat{A}$ and also $\bar{A}$ as $A$ simply. Since we are interested only in the mass ratios and mixings, we use dimensionless expressions $\Phi_{0}=\operatorname{diag}\left(x_{1}, x_{2}, x_{3}\right)$ (with $\left.x_{1}^{2}+x_{2}^{2}+x_{3}^{2}=1\right), P=\operatorname{diag}\left(e^{i \phi_{1}}, e^{i \phi_{2}}, 1\right)$, and $E=\mathbf{1}=\operatorname{diag}(1,1,1)$. Therefore, the parameters $a_{e}, a_{\nu}, a_{u}, a_{d}, \xi_{\nu}, \xi_{u}$, and $\xi_{d}$ are re-defined by Eqs.(3.1)-(3.4).

In the phase matrix $P$ defined by Eq.(1.10), physical values are only differences among $\left(\phi_{1}, \phi_{2}, \phi_{3}\right)$, so that we can take one of $\phi_{i}(i=1,2,3)$ as zero in the parameter fitting for $V_{C K M}$. In this paper, we put $\phi_{3}=0$, so that free parameters are $\left(\phi_{1}, \phi_{2}\right)$. Note that, as we stated in Sec.2.2, $P$ and $P^{*}$ in Eq.(3.1) do not affect $Y_{e}$ practically, because $\Phi_{0}$ and $Y_{e}$ are diagonal, so that $P$ and $P^{*}$ are commutable with $\Phi_{0}$ and $Y_{e}$.

Therefore, in the present model shown in Eqs.(3.1) - (3.5), except for the parameters $\left(x_{1}, x_{2}, x_{3}\right)$, we have 10 adjustable parameters, $\left(a_{\nu}, \alpha_{\nu}, \xi_{\nu}\right),\left(a_{u}, \xi_{u}\right),\left(a_{d}, \alpha_{d}, \xi_{d}\right)$, and $\left(\phi_{1}, \phi_{2}\right)$ for the 16 observable quantities (6 mass ratios in the up-quark, down-quark, and neutrino sectors, four CKM mixing parameters, and $4+2$ PMNS mixing parameters). Especially, quark mass matrices $M_{u}=Y_{u}$ and $M_{d}=Y_{d}$ are fixed by two parameters $\left(a_{u}, \xi_{u}\right)$ and $\left(a_{d}, \alpha_{d}, \xi_{d}\right)$, respectively. Note that those parameters are family number-independent parameters. Therefore, in order to fix those parameters, we use two inputs values, up-quark mass ratios $\left(m_{u} / m_{c}, m_{c} / m_{t}\right)$ and down-quark mass ratios $\left(m_{d} / m_{s}, m_{s} / m_{b}\right)$, respectively, as we discuss in the next subsection 3.2. After the parameters $\left(a_{u}, \xi_{u}\right)$ and $\left(a_{d}, \alpha_{d}, \xi_{d}\right)$ have been fixed by the observed quark mass rations, we have five parameters $\left(a_{\nu}, \alpha_{\nu}, \xi_{\nu}\right)$ and $\left(\phi_{1}, \phi_{2}\right)$ as remaining free parameters. Processes for fitting those five parameters are listed in Table 2. In subsection 3.3, we discuss the fitting of four CKM mixing parameters, $\left|V_{u s}\right|,\left|V_{c b}\right|,\left|V_{u b}\right|$ and $\left|V_{t d}\right|$, by adjusting two parameters $\left(\phi_{1}, \phi_{2}\right)$. 
Table 2: Process for fitting parameters. $N_{\text {parameter }}$ and $N_{\text {input }}$ denote a number of free parameters in the model and a number of observed values which are used as inputs in order to fix these free parameters, respectively. $\sum N_{\ldots}$ means $\sum N_{\text {parameter }}$ or $\sum N_{\text {input }}$

\begin{tabular}{|c|c|c|c|c|c|}
\hline Step & Inputs & $N_{\text {input }}$ & Parameters & $N_{\text {parameter }}$ & Predictions \\
\hline \multirow[t]{2}{*}{$1 \mathrm{st}$} & $m_{u} / m_{c}, m_{c} / m_{t}$ & 2 & $a_{u}, \xi_{u}$ & 2 & 一 \\
\hline & $m_{d} / m_{s}, m_{s} / m_{b}$ & 2 & $a_{d}, \alpha_{d}, \xi_{d}$ & 3 & - \\
\hline 2nd & $\left|V_{c b}\right|,\left|V_{u b}\right|$ & 2 & $\left(\phi_{1}, \phi_{2}\right)$ & 2 & $\left|V_{u s}\right|,\left|V_{t d}\right|, \delta_{C P}^{q}$ \\
\hline $3 \mathrm{rd}$ & $\sin ^{2} 2 \theta_{12}, \sin ^{2} 2 \theta_{23}, R_{\nu}$ & 3 & $a_{\nu}, \alpha_{\nu}, \xi_{\nu}$ & 3 & $\sin ^{2} 2 \theta_{13}, \delta_{C P}^{\ell}$ \\
\hline option & $\Delta m_{32}^{2}$ & & $m_{\nu 3}$ & & $\begin{array}{l}2 \text { Majorana phases, } \frac{m_{\nu 1}}{m_{\nu 2}}, \frac{m_{\nu 2}}{m_{\nu 3}} \\
\quad\left(m_{\nu 1}, m_{\nu 2}, m_{\nu 3}\right),\langle m\rangle\end{array}$ \\
\hline$\sum N_{\ldots}$ & & 9 & & 10 & \\
\hline
\end{tabular}

Also, in subsection 3.4, we do the fitting of PMNS mixing $\left(\sin ^{2} 2 \theta_{12}, \sin ^{2} 2 \theta_{23}\right.$, and $\left.\sin ^{2} 2 \theta_{13}\right)$ and neutrino mass ratio $\left(R_{\nu} \equiv \Delta m_{21}^{2} / \Delta m_{32}^{2}\right)$ by adjusting three parameters $\left(a_{\nu}, \alpha_{\nu}, \xi_{\nu}\right)$.

\subsection{Quark mass ratios}

First let us fix values of $\left(a_{u}, \xi_{u}\right)$ from the up-quark mass ratios. The observed values of the up-quark masses at $\mu=m_{Z}[13]$ are

$$
r_{12}^{u} \equiv \sqrt{\frac{m_{u}}{m_{c}}}=0.045_{-0.010}^{+0.013}, \quad r_{23}^{u} \equiv \sqrt{\frac{m_{c}}{m_{t}}}=0.060 \pm 0.005
$$

We obtain four solutions of $\left(a_{u}, \xi_{u}\right)$ which can give the values (3.6). Among them only one solution

$$
\left(a_{u}, \xi_{u}\right)=(-1.4715,-0.001521),
$$

can give a reasonable prediction of the PMNS mixing as we discuss later.

Secondly, let us fix values of $\left(a_{d}, \alpha_{d}, \xi_{d}\right)$ from the down-quark mass ratios. From the observed down-quark mass ratios 13

$$
r_{12}^{d} \equiv \frac{m_{d}}{m_{s}}=0.053_{-0.003}^{+0.005}, \quad r_{23}^{d} \equiv \frac{m_{s}}{m_{b}}=0.019 \pm 0.006
$$

or [14]

$$
r_{12}^{d} \equiv \frac{m_{d}}{m_{s}}=0.050_{-0.001}^{+0.002}, \quad r_{23}^{d} \equiv \frac{m_{s}}{m_{b}}=0.031 \pm 0.005
$$

we determine the parameters $\left(a_{d}, \xi_{d}, \alpha_{d}\right)$ as follows:

$$
\left(a_{d}, \alpha_{d}, \xi_{d}\right)=\left(-1.4733,15.694^{\circ},+0.004015\right),
$$


which leads to the numerical results as follows: $r_{12}^{d}=0.0612, r_{23}^{d}=0.0312$. These values are inconsistent with the observed values (3.9), but, roughly speaking, those are consistent with (3.10). We think that the light quark mass values are still controversial.

Here, we have tried to fix the parameters $\left(a_{d}, \alpha_{d}, \xi_{d}\right)$ in the down-quark sector by using input parameters [13] for $r_{12}^{d}$ and $r_{23}^{d}$. However, since we have three parameters for two input values $m_{d} / m_{s}$ and $m_{s} / m_{b}$, we cannot fix our three parameters. It is more embarrassing that there is no solution of $m_{s} / m_{b} \sim 0.019$ in the $\left(a_{d}, \alpha_{d}, \xi_{d}\right)$ parameter region. Nevertheless, we found that the minimal value of $m_{s} / m_{b}$ is $m_{s} / m_{b} \sim 0.03$ at $\left(a_{d}, \alpha_{d}, \xi_{d}\right) \sim\left(-1.47,16^{\circ}, 0.004\right)$ which can give a reasonable value of $m_{d} / m_{s}$ at the same time too. Therefore, we take the values in Eq. (3.10), which leads to $r_{12}^{d}=0.0612$ and $r_{23}^{d}=0.0312$. Note that the value $r_{23}^{d}=0.0312$ is considerably large compared with $r_{23}^{d} \simeq 0.019$ by Xing et al. [13], while the value is consistent with $r_{23}^{d} \simeq 0.031$ by Fusaoka and Koide [14]. The values $m_{d}(\mu)$ and $m_{s}(\mu)$ are estimated at a lower energy scale, $\mu \sim 1 \mathrm{GeV}$, so that we consider that the ratio $r_{12}^{d}$ at $\mu=M_{Z}$ is reliable. On the other hand, the value $m_{b}(\mu)$ is extracted at a different energy scale $\mu \sim 4 \mathrm{GeV}$ from $\mu \sim 1$ $\mathrm{GeV}$, so that the value $m_{b}\left(M_{Z}\right)$ is affected by the prescription of threshold effects at $\mu=m_{t}$, while the value $m_{s}\left(M_{Z}\right)$ affected by those at $\mu=m_{c}, \mu=m_{b}$ and $\mu=m_{t}$. We consider that

as for the ratio $r_{23}^{d}$ at $\mu=M_{Z}$ the value is still controversial. Anyhow, we have fixed three parameters $\left(a_{d}, \alpha_{d}, \xi_{d}\right)$ only from two values $m_{d} / m_{s}$ and $m_{s} / m_{b}$.

\subsection{CKM mixing}

Next, we discuss CKM quark mixing. Since the parameters $\left(a_{u}, \xi_{u}\right)$ and $\left(a_{d}, \alpha_{d}, \xi_{d}\right)$ have been fixed by the observed quark mass rations, the CKM mixing matrix elements $\left|V_{u s}\right|,\left|V_{c b}\right|$, $\left|V_{u b}\right|$, and $\left|V_{t d}\right|$ are functions of the remaining two parameters $\phi_{1}$ and $\phi_{2}$. In Fig. 2, with taking $\xi_{u}=-0.001521, a_{u}=-1.4715, a_{d}=-1.47312, \alpha_{d}=15.7^{\circ}$, and $\xi_{d}=0.004091$, we draw allowed regions in the $\left(\phi_{1}, \phi_{2}\right)$ parameter plane which are obtained from the observed values [15] of the CKM mixing matrix elements and the observed value [16] of the $C P$ violating phase parameter $\delta_{C P}^{q}$ in the standard expression of $V_{C K M}$ given by,

$$
\begin{aligned}
& \left|V_{u s}\right|=0.22536 \pm 0.00061 \\
& \left|V_{c b}\right|=0.0414 \pm 0.0012 \\
& \left|V_{u b}\right|=0.00355 \pm 0.00015 \\
& \left|V_{t d}\right|=0.00886_{-0.00033}^{+0.00033} \\
& \delta_{C P}^{q}=69.4^{\circ} \pm 3.4^{\circ}
\end{aligned}
$$

Here, in order to fix the values of $\left(\phi_{1}, \phi_{2}\right)$ we use only two values of the CKM matrix elements as input values in the present analysis, so that the remaining tree are our predictions as references.

As shown in Fig. 1, all the experimental constraints on CKM parameters are satisfied by 


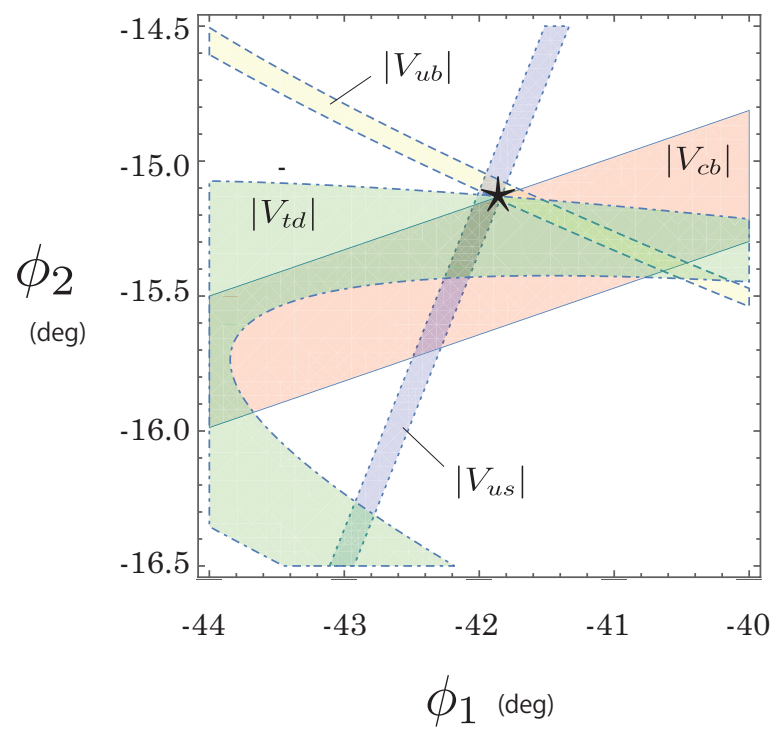

Figure 1: Allowed region in the $\left(\phi_{1}, \phi_{2}\right)$ parameter plane obtained by the observed values of the CKM mixing matrix elements $\left|V_{u s}\right|,\left|V_{c b}\right|,\left|V_{u b}\right|$, and $\left|V_{t d}\right|$. We draw allowed regions obtained from the observed constraints of the CKM mixing matrix elements shown in Eq. (3.11), with taking $\left(a_{u}, \xi_{u}\right)=(-1.4715,-0.001521)$ and $\left(a_{d}, \alpha_{d}, \xi_{d}\right)=\left(-1.4733,15.694^{\circ},+0.004015\right)$. We find that the parameter set around $\left(\phi_{1}, \phi_{2}\right)=\left(-41.815^{\circ},-15.128^{\circ}\right)$ indicated by a star $(\star)$ is consistent with all the observed values.

fine tuning the parameters $\phi_{1}$ and $\phi_{2}$ as

$$
\left(\phi_{1}, \phi_{2}\right)=\left(-41.815^{\circ},-15.128^{\circ}\right)
$$

which leads to the predicted values for the CKM mixing matrix elements and the $C P$ violating phase parameter $\delta_{C P}^{q}$ as follows:

$$
\begin{aligned}
& \left|V_{u s}\right|=0.2261 \\
& \left|V_{c b}\right|=0.0426 \\
& \left|V_{u b}\right|=0.00360 \\
& \left|V_{t d}\right|=0.00920 \\
& \delta_{C P}^{q}=72.4^{\circ} .
\end{aligned}
$$

In spite of our aim described in the Sec. 1, we are forced to introduce family numberdependent parameters $\left(\phi_{1}, \phi_{2}\right)$ in the present model, too, as the same as in the previous model [10]. However, our aim was to describe all the masses and mixing of quarks and leptons in terms of family number-independent parameters expect for the charged lepton masses. Therefore, the introduction of the phase parameters $\phi_{i}$ were against our aim and unwelcome as it is. In Sec.4, we will try to denote these phase parameters $\phi_{i}$ in terms of the observed charged lepton masses $m_{e i}$. 


\subsection{PMNS mixing}

Now let us discuss the PMNS lepton mixing. We have already fixed the four parameters $a_{u}, \xi_{u}, \phi_{1}$ and $\phi_{2}$ as Eqs. (3.7) and (3.12). The remaining free parameters in the neutrino sector are only $\left(a_{\nu}, \alpha_{\nu}, \xi_{\nu}\right)$. We determine the parameter values of $\left(a_{\nu}, \alpha_{\nu}, \xi_{\nu}\right)$ as follows:

$$
\left(a_{\nu}, \alpha_{\nu}, \xi_{\nu}\right)=\left(-2.59,-27.3^{\circ},-0.0115\right)
$$

which are obtained so as to reproduce the observed values [15] of the following PMNS mixing angles and $R_{\nu}$,

$$
\begin{gathered}
\sin ^{2} 2 \theta_{12}=0.846 \pm 0.021, \quad \sin ^{2} 2 \theta_{23}>0.981, \quad \sin ^{2} 2 \theta_{13}=0.093 \pm 0.008 \\
R_{\nu} \equiv \frac{\Delta m_{21}^{2}}{\Delta m_{32}^{2}}=\frac{m_{\nu 2}^{2}-m_{\nu 1}^{2}}{m_{\nu 3}^{2}-m_{\nu 2}^{2}}=\frac{(7.53 \pm 0.18) \times 10^{-5} \mathrm{eV}^{2}}{(2.44 \pm 0.06) \times 10^{-3} \mathrm{eV}^{2}}=(3.09 \pm 0.15) \times 10^{-2}
\end{gathered}
$$

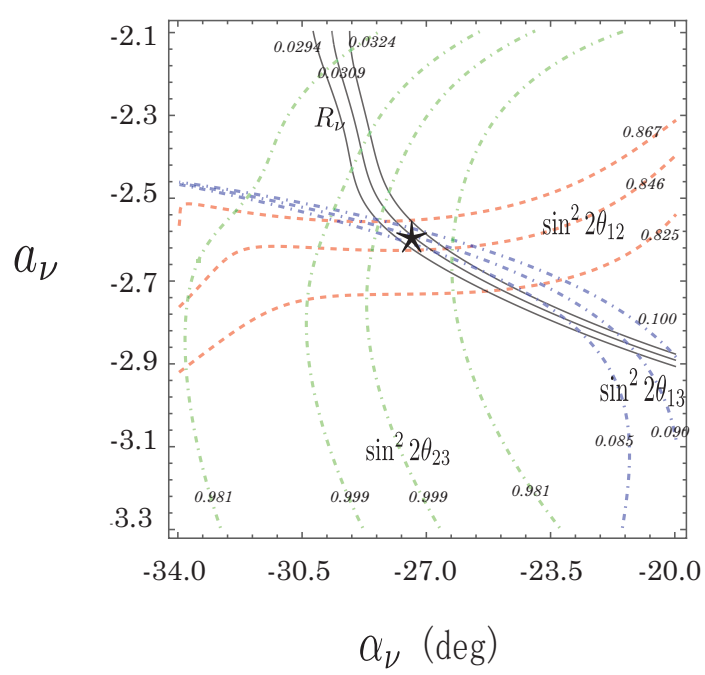

Figure 2: Contour curves of the center, upper, and lower values of the observed PMNS mixing parameters $\sin ^{2} 2 \theta_{12}, \sin ^{2} 2 \theta_{23}, \sin ^{2} 2 \theta_{13}$, and $R_{\nu}$ in the $\left(a_{\nu}, \alpha_{\nu}\right)$ parameter space. We draw the curves for the case of $\xi_{\nu}=-0.0115$ and $\left(\phi_{1}, \phi_{2}\right)=\left(-41.815^{\circ},-15.128^{\circ}\right)$ with taking $\left(a_{u}, \xi_{u}\right)=$ $(-1.4715,-0.001521)$. We find that the parameter set around $\left(a_{\nu}, \alpha_{\nu}\right)=\left(-2.59,-27.3^{\circ}\right)$ indicated by a star $(\star)$ is consistent with all the observed values.

In Fig. 2, we show the contour plots of the observed PMNS mixing parameters $\sin ^{2} 2 \theta_{12}$, $\sin ^{2} 2 \theta_{23}, \sin ^{2} 2 \theta_{13}$, and $R_{\nu}$ in the $\left(a_{\nu}, \alpha_{\nu}\right)$ parameter space for the case of $\xi_{\nu}=-0.0115$ with taking $\left(\phi_{1}, \phi_{2}\right)=\left(-41.815^{\circ},-15.128^{\circ}\right)$ and $\left(a_{u}, \xi_{u}\right)=(-1.4715,-0.001521)$. It is found that all the PMNS mixing parameters are well consistent with the observe values in Eqs. (3.15) and (3.16). As shown in Fig. 2, all the experimental constraints on the PMNS mixing parameters 
are satisfied by fine tuning the parameters $a_{\nu}, \alpha_{\nu}$, and $\xi_{\nu}$ as

$$
\left(a_{\nu}, \alpha_{\nu}, \xi_{\nu}\right)=\left(-2.59,-27.3^{\circ},-0.0115\right)
$$

which leads to the predicted values for the PMNS mixing angles, $R_{\nu}$, and the Dirac $C P$ violating phase parameter $\delta_{C P}^{\ell}$ in the standard expression of $U_{P M N S}$ as follows:

$$
\begin{aligned}
& \sin ^{2} 2 \theta_{12}=0.857, \\
& \sin ^{2} 2 \theta_{23}=0.993, \\
& \sin ^{2} 2 \theta_{13}=0.0964, \\
& R_{\nu}=0.0316, \\
& \delta_{C P}^{\ell}=-76.0^{\circ} .
\end{aligned}
$$

Our model predicts $\delta_{C P}^{\ell}=-76.0^{\circ}$ for the Dirac $C P$ violating phase in the lepton sector. This is very interesting because the value shows a size similar to $\delta_{C P}^{q}=+72.4^{\circ}$ in the CKM mixing matrix.

\subsection{Neutrino masses}

We can predict neutrino masses, for the parameters given by (3.7), (3.12), and (3.14), as follows

$$
m_{\nu 1} \simeq 0.00046 \mathrm{eV}, \quad m_{\nu 2} \simeq 0.00879 \mathrm{eV}, \quad m_{\nu 3} \simeq 0.0502 \mathrm{eV},
$$

by using the input value [15] $\Delta m_{32}^{2} \simeq 0.00244 \mathrm{eV}^{2}$.

We also predict the effective Majorana neutrino mass [17] $\langle m\rangle$ in the neutrinoless double beta decay as

$$
\langle m\rangle=\left|m_{\nu 1}\left(U_{e 1}\right)^{2}+m_{\nu 2}\left(U_{e 2}\right)^{2}+m_{\nu 3}\left(U_{e 3}\right)^{2}\right| \simeq 3.8 \times 10^{-3} \mathrm{eV}
$$

In Table 3, we list our predictions of the CKM and the PMNS mixing parameters and quark mass ratios and neutrino masses together with the observed values .

\section{VEV relation between $P$ and $\Phi_{0}$}

So far, we have tried to described all Yukawaon VEV matrices $\left\langle\hat{Y}_{f}\right\rangle$ by using only the observed charged lepton masses $m_{e i}$ as input values. We have also tried to understand $C P$ violating phase only by using phase parameters $\alpha_{f}$ which are phases of family number-independent parameters $a_{f}$. Nevertheless, all such attempts have failed because we always needed a phase matrix $P$ in order to fit reasonable CKM mixing and quark mass ratios. In this paper, we accept the existence of $P$, and we try to understand the values of the phase parameters $\phi_{i}$ in $P$ from the charged lepton mass values $m_{e i}$.

In the present model, we have flavon VEVs with diagonal form, $P, \bar{P}, E, \bar{E}, \Phi_{0}, \bar{\Phi}_{0}, \Phi_{e}$, $\bar{\Phi}_{e}$, and $\hat{Y}_{e}$. (Here, we omit "〈" and "〉".) In considering combinations of $\mathrm{U}(3) \mathbf{8}+\mathbf{1}$ scalars 
Table 3: Predicted values vs. observed values.

\begin{tabular}{|c|ccccccccc|}
\hline & $\left|V_{u s}\right|$ & $\left|V_{c b}\right|$ & $\left|V_{u b}\right|$ & $\left|V_{t d}\right|$ & $\delta_{C P}^{q}$ & $r_{12}^{u}$ & $r_{23}^{u}$ & $r_{12}^{d}$ & $r_{23}^{d}$ \\
\hline Pred & 0.2261 & 0.0426 & 0.00360 & 0.00920 & $72.4^{\circ}$ & 0.0458 & 0.0600 & 0.0611 & 0.0312 \\
Obs & 0.22536 & 0.0414 & 0.00355 & 0.00886 & $69.4^{\circ}$ & 0.045 & 0.060 & 0.053 & 0.019 \\
& \pm 0.00061 & \pm 0.0012 & \pm 0.00015 & ${ }_{-0.00032}^{+0.00033}$ & $\pm 3.4^{\circ}$ & ${ }_{-0.010}^{+0.013}$ & \pm 0.005 & ${ }_{-0.003}^{+0.005}$ & ${ }_{-0.006}^{+0.006}$ \\
\hline & $\sin ^{2} 2 \theta_{12}$ & $\sin ^{2} 2 \theta_{23}$ & $\sin ^{2} 2 \theta_{13}$ & $R_{\nu}\left[10^{-2}\right]$ & $\delta_{C P}^{\ell}$ & $m_{\nu 1}[\mathrm{eV}]$ & $m_{\nu 2}[\mathrm{eV}]$ & $m_{\nu 3}[\mathrm{eV}]$ & $\langle m\rangle[\mathrm{eV}]$ \\
\hline Pred & 0.857 & 0.993 & 0.0964 & 3.16 & $-76.0^{\circ}$ & 0.00046 & 0.00879 & 0.0502 & 0.00377 \\
Obs & 0.846 & 0.999 & 0.093 & 3.09 & - & - & - & - & $<\mathrm{O}\left(10^{-1}\right)$ \\
& \pm 0.021 & ${ }_{-0.018}^{+0.001}$ & \pm 0.008 & \pm 0.15 & & & & & - \\
\hline
\end{tabular}

out of those flavons, we have to consider a combination without the parameter $\Delta$ for $E$ and $P$ because the $R$ charges of $\Phi_{0}$ and $\Phi_{e}$ do not contain the parameter $\Delta$. Only a combination with $P$ whose $R$ charge does not include the parameter $\Delta$ is

$$
(P \bar{E}+E \bar{P})_{i}^{j}=\delta_{i}^{j}\left(e^{i \phi_{i}}+e^{-i \phi_{i}}\right)=\delta_{i}^{j} 2 \cos \phi_{i},
$$

with $R$ charge of $R=\frac{1}{2}(1+\Delta)+\frac{1}{2}(1-\Delta)=+1$. On the other hand, since we have $R$ charges

$$
R\left(\Phi_{e}\right)=\frac{1}{2}, \quad R\left(\Phi_{0}\right)=\frac{1}{2},
$$

for $\Phi_{e}$ and $\Phi_{0}$ as discussed in Sec.2.3, we have only two combinations which have $R$ charge of $R=+1,\left(\Phi_{e}\right)_{i k}\left(\bar{\Phi}_{e}\right)^{k j}$ and $\left(\Phi_{0}\right)_{i \alpha}\left(\bar{\Phi}_{0}\right)^{\alpha j}$. [Note that $\left(\Phi_{0} \bar{\Phi}_{e}+\Phi_{e} \bar{\Phi}_{0}\right)$ cannot be a candidate, because it has $R=+1$ but it is not a $\mathrm{U}(3)^{\prime}$ singlet.] Therefore, we can take superpotential

$$
W=\lambda_{1}\left[(P \bar{E}+E \bar{P}) \hat{\Theta}_{P}\right]+\lambda_{2}\left[\left(\Phi_{e} \bar{\Phi}_{e}+b \Phi_{0} \bar{\Phi}_{0}\right) \hat{\Theta}_{P}\right]
$$

so that we obtain

$$
k(P \bar{E}+E \bar{P})=\Phi_{e} \bar{\Phi}_{e}+b \Phi_{0} \bar{\Phi}_{0}
$$

i.e.

$$
2 k \cos \phi_{i}=x_{i}^{4}+b x_{i}^{2},
$$

where we have used the dimensionless expressions of $P, E, \Phi_{0}$ and $\Phi_{e}$, Eq.(2.9), Eq.(1.7) with $v_{0}=1$, and so on.

Eliminating the coefficient $k$ in Eq.(4.5), we obtain two equations

$$
\begin{aligned}
& \frac{\cos \phi_{1}}{\cos \phi_{3}}=\frac{x_{1}^{4}+b x_{1}^{2}}{x_{3}^{4}+b x_{3}^{2}}, \\
& \frac{\cos \phi_{2}}{\cos \phi_{3}}=\frac{x_{2}^{4}+b x_{2}^{2}}{x_{3}^{4}+b x_{3}^{2}} .
\end{aligned}
$$


In Sec.3, we have obtained numerical results $\phi_{1}=-41.815^{\circ}$ and $\phi_{2}=-15.128^{\circ}$ by putting $\phi_{3}$ as $\phi_{3}=0$. In order to avoid confusing, we use notation $\tilde{\phi}_{i}$ for these numerical results of $\phi_{i}$. Since we can choose any value of $\phi_{0}$ in $\phi_{i} \rightarrow \phi_{i}+\phi_{0}$, we define $\phi_{i}$ in Eq.(4.5) as

$$
\phi_{1}=\phi_{0}+\tilde{\phi}_{1}, \quad \phi_{2}=\phi_{0}+\tilde{\phi}_{2}, \quad \phi_{3}=\phi_{0} .
$$

The equations (4.6) and (4.7) have two unknown parameters $\phi_{0}$ and $b$ under the input values $\tilde{\phi}_{1}$ and $\tilde{\phi}_{2}$. So, we obtain

$$
\phi_{0}=-45.903^{\circ}, \quad b=-1.11586,
$$

which means

$$
\phi_{1}=-87.718^{\circ}, \quad \phi_{2}=-61.031^{\circ}, \quad \phi_{3}=-45.903^{\circ} .
$$

Regrettably, since we need two input parameters $\phi_{0}$ and $b$ in order to predict the values $\tilde{\phi}_{1}$ and $\tilde{\phi}_{2}$, the present model has no predictability for phase parameters $\left(\phi_{1}, \phi_{2}, \phi_{3}\right)$. (If we use the fitting value $\tilde{\phi}_{1}=-41.815^{\circ}$ as input value in addition to the input value $b=-1.11586$, we can predict the value $\tilde{\phi}_{2}$ together with the value of $\phi_{0}$.) However, note that the parameters $\phi_{i}$ are family number-dependent parameters, while the parameters $\phi_{0}$ and $b$ are family numberindependent parameters. Therefore, the aim of the Yukawaon model that we understand mass spectra and mixings of all quarks and leptons only in terms of charged lepton mass spectrum and without using any other family number-dependent parameters has been achieved in this scenario.

\section{Concluding remarks}

We have tried to describe quark and lepton mass matrices by using only the observed values of charged lepton masses $\left(m_{e}, m_{\mu}, m_{\tau}\right)$ as input parameters with family number-dependent values. Namely, we have investigated whether we can describe all other observed mass spectra (quark and neutrino mass spectra) and the quark- and lepton-mixings (the CKM and the PMNS mixings) without using any other family number-dependent parameters. In conclusion, as seen in Sec.3, we have obtained reasonable results. Our predicted values are listed in Table 3.

As seen in Sec.3, we have still used the phase matrix $P$ defined by Eq.(1.10) in order to fit the observed CKM mixing parameters similarly to the past Yukawaon models. However, as seen in Sec.4, the most remarkable point of the present paper is that we have succeeded in describing the family number-dependent parameters $\left(\phi_{1}, \phi_{2}, \phi_{3}\right)$ by the family number-independent parameters $\phi_{0}$ and $b$. Therefore, the main aim in the Yukawaon model have been achieved in the present work. However, regrettably, the mechanism proposed has no predictability of the phase parameters $\phi_{i}$, although it transforms unwelcome family number-dependent parameters into family number-independent parameters. The mechanism will be improved in a future version.

The successful results in the present work suggests the following items: (i) the flavor basis in which the charged lepton mass matrix $M_{e}$ is diagonal is more fundamental basis in the flavor 
physics. (ii) The parameters $\left(m_{e}, m_{\mu}, m_{\tau}\right)$ (i.e. $\left(x_{1}, x_{2}, x_{3}\right)$ defined by Eq.(1.7)) are fundamental parameters in quark and lepton physics. Note that the parameter values $\left(m_{e}, m_{\mu}, m_{\tau}\right)$ are extremely hierarchical, while the parameter values $\left(x_{1}, x_{2}, x_{3}\right)$ are mildly hierarchical. Understanding of the values of $\left(x_{1}, x_{2}, x_{3}\right)$ will be left to our next task in future. Then, the relation $\left(m_{e}+m_{\mu}+m_{\tau}\right) /\left(\sqrt{m_{e}}+\sqrt{m_{\mu}}+\sqrt{m_{\tau}}\right)^{2}=2 / 3$ [18] may play an essential role in investigating the origin of the parameter values $\left(x_{1}, x_{2}, x_{3}\right)$. For reference, we give a trial model on the charged lepton mass relation within the framework of the present Yukawaon model in Appendices A and $\mathrm{B}$, although this is only a trial one.

In this model, there are four phase parameters $\alpha_{\nu}, \alpha_{d}$ and $\left(\tilde{\phi}_{1}, \tilde{\phi}_{2}\right)$. The parameters $\alpha_{\nu}$ and $\alpha_{d}$ play a role in giving mass ratios in the neutrino and down-quark sectors, respectively. The parameters which purely contribute to the CKM and PMNS mixing matrices as $C P$ violating phase parameters are only $\left(\tilde{\phi}_{1}, \tilde{\phi}_{2}\right)$. These parameters can commonly contribute to CKM and PMNS mixing matrix, so that those play an essential role in both the predicted values of $\delta_{C P}^{q}$ and $\delta_{C P}^{\ell}$. It is interesting that, in spite of different values between $\alpha_{d}$ and $\alpha_{\nu}$, the results of $C P$ violating parameters $\delta_{C P}^{q}$ and $\delta_{C P}^{\ell}$ take a similar magnitude, $\delta_{C P}^{q} \sim-\delta_{C P}^{\ell} \sim 70^{\circ}$.

In conclusion, it seems to be certain that all of the observed hierarchical structures of quark and lepton masses and mixings are commonly originated from the hierarchical values of $\left(m_{e}, m_{\mu}, m_{\tau}\right)$ which are described by the fundamental parameters $\left(x_{1}, x_{2}, x_{3}\right)$. Of course, the present Yukawaon model has to be still improved with respect to the $R$ charge assignments, number of flavons, number of adjustable parameters, $C P$ violating phase parameters, and so on. In addition to this, our next task is to investigate the origin of the parameters $\left(x_{1}, x_{2}, x_{3}\right)$. 


\section{Appendix A: Charged Lepton Mass Relation in the Yukawaon Model}

The charged lepton mass relation [18]

$$
K \equiv \frac{m_{e}+m_{\mu}+m_{\tau}}{\left(\sqrt{m_{e}}+\sqrt{m_{\mu}}+\sqrt{m_{\tau}}\right)^{2}}=\frac{2}{3}
$$

is one of the main motives of the Yukawaon model in the earlier stage [19]. The relation (A.1) can be understood from VEV of U(3) $8+\mathbf{1}$ scalar, $\left\langle\hat{\Phi}_{e}\right\rangle=\operatorname{diag}\left(\sqrt{m_{e}}, \sqrt{m_{\mu}}, \sqrt{m \tau}\right)$ as

$$
K=\frac{\operatorname{Tr}\left[\hat{\Phi}_{e} \hat{\Phi}_{e}\right]}{\left(\operatorname{Tr}\left[\hat{\Phi}_{e}\right]\right)^{2}}
$$

where we have omitted VEV notation "〈" and "〉" for simplicity. Also, hereafter, for simplicity, we denote $\operatorname{Tr}[A]$ as $[A]$. However, in the present scenario of the Yukawaon model, there is no $\mathbf{8}+\mathbf{1}$ scalar $\hat{\Phi}_{e}$, but we have only $\mathbf{6}$ and $\mathbf{6}^{*}$ scalars $\Phi_{e}$ and $\bar{\Phi}_{e}$. The purpose of the present paper is to understand mass ratios and mixings of quarks and leptons under the given parameters $\left(m_{e}, m_{\mu}, m_{\tau}\right)$, and it is not to investigate that the origin of the values $\left(m_{e}, m_{\mu}, m_{\tau}\right)$.

However, in this Appendix, let us try to understand the mass relation (A.1) according to an idea suggested in Ref.[19]. First, let us introduce $\mathbf{8}+\mathbf{1}$ scalar $\hat{\Phi}_{e}$. By using the following superpotential:

$$
W=\mu\left[\hat{\Phi}_{e} \hat{\Theta}_{e}\right]+\lambda_{e}\left[\left(\Phi_{e} \bar{E}+E \bar{\Phi}_{e}\right) \hat{\Theta}_{e}\right]
$$

we obtain a relation

$$
\hat{\Phi}_{e}=\Phi_{e} \bar{E}+E \bar{\Phi}_{e}
$$

Since $R(E)=\frac{1}{2}(1-\Delta)$ as seen in Eq.(2.19), $\hat{\Phi}_{e}$ has $R$ charge as

$$
R\left(\hat{\Phi}_{e}\right)=1-\frac{1}{2} \Delta
$$

Let us take $\Delta=+1$, so that we have

$$
R\left(\hat{\Phi}_{e}\right)=R\left(\Phi_{e}\right)=\frac{1}{2}
$$

This choice (A.6) causes no problem because $\hat{\Phi}_{e}$ and $\Phi_{e}$ have different transformation under $\mathrm{U}(3) \times \mathrm{U}(3)^{\prime}$. We will comment on the choice $R(E)=0$ later.

Since $R\left(\hat{\Phi}_{e}\right)=1 / 2$, we assume the following superpotential

$$
\left.W=\frac{1}{\Lambda}\left(\lambda\left[\hat{\Phi}_{e} \hat{\Phi}_{e}\right]^{2}+\lambda^{\prime}\left[\hat{\Phi}_{e}\right]\right)^{2}\left[\hat{\Phi}_{8} \hat{\Phi}_{8}\right]\right)
$$


where $\hat{\Phi}_{8}$ is an octet part of the nonet $\hat{\Phi}_{e}$ defined by

$$
\hat{\Phi}_{8} \equiv \hat{\Phi}_{e}-\frac{1}{3}\left[\hat{\Phi}_{e}\right] \mathbf{1}
$$

The first term in Eq.(A.7) is the conventional nonet-nonet term. The second term is an (octet-octet) $\times($ singlet-singlet) interaction term [19] although the second term is still $\mathrm{SU}(3)$ invariant. In order to derivate the relation (A.1), the assumption of the second term is essential. By noticing that the second term can be expressed as

$$
\left[\hat{\Phi}_{e} \hat{\Phi}_{e}\right]\left[\hat{\Phi}_{e}\right]^{2}-\frac{1}{3}\left[\hat{\Phi}_{e}\right]^{4}
$$

we obtain

$$
\frac{\partial W}{\partial \hat{\Phi}_{e}}=\frac{1}{\Lambda}\left\{2\left(2 \lambda\left[\hat{\Phi}_{e} \hat{\Phi}_{e}\right]+\lambda^{\prime}\left[\hat{\Phi}_{e}\right]^{2}\right) \hat{\Phi}_{e}+2 \lambda^{\prime}\left(\left[\hat{\Phi}_{e} \hat{\Phi}_{e}\right]-\frac{2}{3}\left[\hat{\Phi}_{e}\right]^{2}\right)\left[\hat{\Phi}_{e}\right] \mathbf{1}\right\} .
$$

The coefficients of $\hat{\Phi}_{e}$ and $\mathbf{1}$ must be zero in order to have a nontrivial solution of $\hat{\Phi}_{e}$ (non-zero and non-unit matrix form). Thus, we demand

$$
2 \lambda\left[\hat{\Phi}_{e} \hat{\Phi}_{e}\right]+\lambda^{\prime}\left[\hat{\Phi}_{e}\right]^{2}=0
$$

and

$$
\left[\hat{\Phi}_{e} \hat{\Phi}_{e}\right]-\frac{2}{3}\left[\hat{\Phi}_{e}\right]^{2}=0
$$

Eq.(A.11) requires a special relation between $\lambda$ and $\lambda^{\prime}$. Note that the relation (A.12) is independent of the explicit value of $\lambda^{\prime}$.

Let us comment on the choice of $\Delta=+1$. This choice means that $R(E)=0$, so that a $\mathrm{U}(3)$ nonet $(E \bar{E})$ takes $R(E \bar{E})=0$. Therefore, the factor $E \bar{E}$ can be inserted into any terms with $R=2$ in the superpotential. However, since $\langle E \bar{E}\rangle=\mathbf{1}$, this does not affect the obtained VEV relations practically. The choice $\Delta=+1$ also gives $R$ charges of $S_{f}$ as

$$
\left(R\left(S_{\nu}\right), R\left(S_{d}\right), R\left(S_{e}\right), R\left(S_{u}\right)\right)=\left(\frac{1}{2}, 1, \frac{3}{2}, 2\right)
$$

It is interesting that the values $(1 / 2,1,3 / 2,2)$ in (A.13) are the same as the values of $\Phi_{f}$ as seen in Eq.(2.30), but the arrangements are different, i.e. $(e, u, \nu, d)$ for $R\left(\Phi_{f}\right)$, while $(\nu, d, e, u)$ for $R\left(S_{f}\right)$. 


\section{Appendix B: Alternative Scenario for Charged Lepton Mass Relation}

In Appendix $\mathrm{A}$, we have introduced the new flavon $\hat{\Phi}_{e}$ in addition to the flavons $\Phi_{e}$ and $\bar{\Phi}_{e}$. So far, we have adhered the idea that the Yukawaon VEV structures take a universal form $\hat{Y}_{f}=\Phi_{f} \bar{\Phi}_{f}+\xi_{f} \mathbf{1}(f=e, \nu, d, u)$. However, if we accept an idea that a structure of $\hat{Y}_{e}$ is exceptional, we can introduce $\hat{\Phi}_{e}$ without introducing $\Phi_{e}$ and $\bar{\Phi}_{e}$ as following

$$
\hat{Y}_{e}=\hat{\Phi}_{e} \hat{\Phi}_{e}, \quad \hat{\Phi}_{e}=\Phi_{0} \bar{\Phi}_{0} .
$$

This expression (B.1) is rather simpler compared with the expression $\hat{Y}_{e}=\Phi_{e} \bar{\Phi}_{e}$ with $\bar{P}_{e} \Phi_{e} \bar{P}_{e}=$ $\bar{\Phi}_{0} S_{e} \bar{\Phi}_{0}$ given in Sec.2. Therefore, in this scenario, without $\Phi_{e}$ and $\bar{\Phi}_{e}$ [i.e. without Eqs.(A.3) (A.6)], we can use (A.7), so that we can obtain the charged lepton mass relation (A.1).

However, in this scenario, since we have $R$ charges

$$
R\left(\hat{Y}_{e}\right)=+1, \quad R\left(\hat{\Phi}_{e}\right)=\frac{1}{2}, \quad R\left(\Phi_{0}\right)=\frac{1}{4},
$$

we cannot put the $\Phi_{0} \bar{\Phi}_{0}$ term in Eq.(4.3). In order to avoid this trouble, in a superpotential for $P \bar{E}+E \bar{P}$, we a little change the scenario in Sec.4. We assume a mechanism similar to Eqs.(2.1) - (2.3):

$$
W_{P}=\left[\left(\lambda_{1}(P \bar{E}+E \bar{P})+\lambda_{2} \hat{\Phi}_{e} \hat{\Phi}_{e}\right) \hat{\Theta}_{P}\right]+\left[\lambda_{1}^{\prime}(P \bar{E}+E \bar{P})+\lambda_{2}^{\prime} \hat{\Phi}_{e} \hat{\Phi}_{e}\right]\left[\hat{\Theta}_{P}\right],
$$

so that we obtain

$$
k(P \bar{E}+E \bar{P})=\hat{\Phi}_{e} \hat{\Phi}_{e}+\xi_{P} \mathbf{1},
$$

i.e.

$$
2 k \cos \phi_{i}=x_{i}^{4}+\xi_{P},
$$

instead of Eqs.(4.3) and(4.4), respectively. From Eqs.(4.5) and (4.6) with $\xi_{P}$ instead of $b$ terms, we obtain numerical solution

$$
\phi_{0}=29.222^{\circ}, \quad \xi_{P}=-5.9619,
$$

so that

$$
\phi_{1}=-12.623^{\circ}, \quad \phi_{2}=14.069^{\circ}, \quad \phi_{3}=29.222^{\circ} .
$$

Maybe, other scenarios are also possible. The purpose in this paper is not to propose a scenario which derives the relation (A.1) but to demonstrate a possibility that the family number-dependent parameters $\left(\phi_{1}, \phi_{2}, \phi_{3}\right)$ can, in principle, be described by a family numberindependent parameter. More reasonable scenario will be given in future. 


\section{References}

[1] K. Akama and H. Terazawa, INS-Report-257 (1976) (INS, University of Tokyo); H. Terazawa, Y. Chikashige, and K. Akama, Phys.Rev. D15, 480 (1977); T. Maehara and T. Yanagida, Prog. Theor. Phys. 60, 822 (1978).

[2] Y. Koide, Phys Rev. D 79, 033009 (2009); Phys. Lett. B 680, 76 (2009).

[3] C. D. Froggatt and H. B. Nelsen, Nucl. Phys. B 147, 277 (1979). For recent works, for instance, see R. N. Mohapatra, AIP Conf. Proc. 1467, 7 (2012); A. J. Buras et al., JHEP 1203 (2012) 088.

[4] P. Minkowski, Phys. Lett. B 67, 421 (1977); M. Gell-Mann, P. Ramond, and R. Slansky, Proceedings of the Supergravity Stony Brook Workshop, New York, 1979, edited by P. Van Nieuwenhuizen and D. Freedman (North-Holland, Amsterdam, 1979); T. Yanagida, Proceedings of the Workshop on Unified Theories and Baryon Number in the Universe, Tsukuba, Japan 1979, edited by A. Sawada and A. Sugamoto [KEK Report No. 79-18, Tsukuba]; R. Mohapatra and G. Senjanovic, Phys. Rev. Lett. 44, 912 (1980).

[5] N. Cabibbo, Phys. Rev. Lett. 10, 531 (1963); M. Kobayashi and T. Maskawa, Prog. Theor. Phys. 49, 652 (1973).

[6] H. Nishiura and Y. Koide, Phys. Rev. D 83, 035010 (2011); Euro. Phys. J. C 72, 1933 (2012); Phys. Lett. B 712, 396 (2012).

[7] P. F. Harrison and W. G. Scott, Phys. Lett. B 535, 163 (2002); Phys. Lett. B 557, 76 (2003); Z.-z. Xing, Phys. Lett. B 533, 85 (2002); E. Ma, Phys. Rev. Lett. 90, 221802 (2003);

C. I. Low and R. R. Volkas, Phys. Rev. D 68, 033007 (2003).

[8] Y. Koide and H. Nishiura, Euro. Phys. J. C 73, 2277 (2013); JHEP 04, 166 (2013); Phys. Rev. D 88, 116004 (2013).

[9] K. Abe et al. (T2K collaboration), Phys. Rev. Lett. 107, 041801 (2011); MINOS collaboration, P. Adamson et. al., Phys. Rev. Lett. 107, 181802 (2011); Y. Abe et al. (DOUBLECHOOZ Collaboration), Phys. Rev. Lett. 108, 131801 (2012); F. P. An, et al. (Daya-Bay collaboration), Phys. Rev. Lett. 108, 171803 (2012); J. K. Ahn, et al. (RENO collaboration), Phys. Rev. Lett. 108, 191802 (2012).

[10] Y. Koide and H. Nishiura, Phys. Rev. D 90, 016009 (2014); Phys. Rev. D 90, 117903 (2014).

[11] Y. Koide and H. Fusaoka, Z. Phys. C 71, 459 (1996). 
[12] B. Pontecorvo, Zh. Eksp. Teor. Fiz. 33, 549 (1957) and 34, 247 (1957); Z. Maki, M. Nakagawa, and S. Sakata, Prog. Theor. Phys. 28, 870 (1962).

[13] Z.-z. Xing, H. Zhang, and S. Zhou, Phys. Rev. D 77, 113016 (2008).

[14] H. Fusaoka and Y. Koide, Phys. Rev. D 57, 3986 (1998).

[15] K. A. Olive et al. (Particle Data Group), Chinese Phys. C, 38, 09001 (2014).

[16] UTfit Collaboration, Fit results: Summer 2014 at http://www.utfit.org/UTfit/ResultsSummer2014PostMoriondSM

[17] M. Doi, T. Kotani, H. Nishiura, K. Okuda, and E. Takasugi, Phys. Lett. B 103, 219 (1981) and B 113, 513 (1982).

[18] Y. Koide, Lett. Nuovo Cim. 34, 201 (1982); Phys. Lett. B 120, 161 (1983); Phys. Rev. D 28, 252(1983).

[19] Y. Koide, Mod. Phys. Lett. A5, 2319 (1990). Also see, Y. Koide, Phys Rev. D 79, 033009 (2009). 